\title{
Effect of compliance with GOLD treatment recommendations on COPD health care resource utilization, cost, and exacerbations among patients with COPD on maintenance therapy
}

Swetha R Palli, MS; Siting Zhou, PhD; Asif Shaikh, MD; and Vincent J Willey, PharmD

\section{What is already known about this subject}

- The Global Initiative for Chronic Obstructive Lung Disease (GOLD) has provided evidence-based recommendations for the treatment of individuals with chronic obstructive pulmonary disease (COPD) for more than 20 years.

- Despite this, previous research has shown suboptimal rates of compliance to the GOLD recommendations with both under- and overprescribing of COPD therapies.

- Suboptimal compliance with the previous GOLD recommendations has been associated with worse clinical and economic COPD outcomes.

\author{
What this study adds \\ - Suboptimal compliance with GOLD \\ treatment recommendations remains \\ problematic, with the primary reason \\ for noncompliance appearing to be \\ overuse of inhaled corticosteroid- \\ containing therapies in those patients \\ prescribed maintenance therapy. \\ - Compliance with GOLD recommenda- \\ tions was associated with significant \\ reductions in COPD-related medical \\ costs, and in particular, COPD-related \\ inpatient utilization and their costs. \\ - In addition, treatment regimens \\ consistent with the GOLD \\ recommendations were associated with \\ moderate reductions in rates of and \\ time to COPD exacerbations.
}

OBJECTIVE: To compare COPD-related health care resource utilization (HRU) and costs, as well as exacerbation rates, among patients with COPD on maintenance therapy based on 2017 GOLD treatment recommendation compliance status per GOLD ABCD risk group classification in a U.S. commercially insured/ Medicare Advantage population.

METHODS: This retrospective cohort study utilized administrative claims data in the HealthCore Integrated Research Database. The COPD population was identified using a previously validated claims-based predictive model. Among this population, patients with $\geq 1$ claim for a COPD maintenance medication (earliest maintenance fill-date $=$ index

\begin{abstract}
Author affiliations
Swetha R Palli, MS, and Asif Shaikh, MD, Boehringer Ingelheim Pharmaceuticals, Ridgefield, CT. Siting Zhou, PhD, and Vincent J Willey, PharmD, HealthCore, Wilmington, DE.
\end{abstract}

AUTHOR CORRESPONDENCE:

Swetha R Palli, 203.798.4460;

swetha.palli@boehringer-ingelheim.com

J Manag Care Spec Pharm. 2021;27(5):625-37

Copyright $\odot 2021$, Academy of Managed Care Pharmacy. All rights reserved.

date) between January 1, 2014, and March 31, 2017, were identified. Patients were required to be aged $\geq 40$ years, have $\geq 12$ months of pre-index and $\geq 30$ days of post-index health plan enrollment, with no diagnosis for asthma, cystic fibrosis, and/ or lung cancer at any time from January 1, 2013, to March 31, 2018. Patients were categorized into exacerbation risk/ symptomatology groups according to the 2017 GOLD ABCD assessment recommendations and were then classified into treatment-compliance status based on their maintenance therapy. Multivariable analyses were conducted to examine post-index COPD-related HRU, costs, and exacerbations by compliance status. 
RESULTS: The primary analytical study sample included 38,382 patients in the GOLD A/B group and 6,525 in the GOLD C/D group. Patients were further categorized into GOLD A $(n=19,345)$, $B(n=19,037), C(n=1,865)$, and $D(n=4,670)$. GOLD-compliant regimens were observed in $32.9 \%$ of patients in the GOLD A/B group and in $58.9 \%$ of patients in the GOLD C/D group. Inhaled corticosteroidcontaining regimens were the most commonly observed noncompliant regimen. Patients on compliant regimens had significantly fewer COPD-related inpatient and emergency department visits and therefore had significantly lower COPD-related medical costs in both the GOLD A/B and C/D cohorts. Similar results were observed for individual GOLD cohorts B, C, and D. These savings were offset by increased pharmacy expenditures. Being on GOLD guidelinecompliant regimens significantly reduced the risk of exacerbation by $8 \%$ (hazard ratio $[\mathrm{HR}]=0.92 ; P<0.0001$ ) in the GOLD A/B cohort and by $12 \%(H R=0.88 ; P=0.0005)$ in the GOLD C/D cohort, and was also associated with a significantly reduced exacerbation rate in the GOLD A/B (rate ratio $[R R]=0.93 ; P<0.0001)$ and GOLD C/D $(R R=0.93$; $P=0.0129)$ groups.

CONCLUSIONS: This study suggests a continuing trend of high prevalence of suboptimal prescriber compliance to GOLD treatment recommendations. Treatment regimens compliant with GOLD recommendations were associated with improvement in exacerbations, reduced COPD-related HRU, and COPD-related medical cost offsets.

Chronic obstructive pulmonary disease (COPD) has significant clinical, societal, and economic effects. ${ }^{1}$ Since its launch in 1998, the Global Initiative for Chronic Obstructive Lung Disease (GOLD) recommendations for COPD management have focused on reducing existing symptom burden, decreasing the risk of future exacerbations, and improving health status. ${ }^{2,3}$ Based on these guiding principles and the current evidence base, GOLD endorses long-acting bronchodilator combinations with differing mechanisms of action including long-acting muscarinic antagonists (LAMA) and long-acting beta2-agonists (LABA) in patients with COPD insufficiently controlled on monobronchodilator therapy. Inhaled corticosteroid (ICS)-containing medications, added to bronchodilator therapy, should be reserved for patients with moderate-to-very severe COPD with further exacerbations or dyspnea or elevated blood eosinophil counts. ${ }^{2,3}$

Despite these recommendations, COPD clinical practice patterns suggest extensive under- and overtreatment., ${ }^{4,5}$ One study reported that $>60 \%$ of patients with COPD from 19 health plans across the United States were not prescribed any COPD maintenance medications (LABA, LAMA, ICS, or methylxanthines). ${ }^{4}$ Others found that ICS-containing medications were overprescribed for patients with less severe COPD. ${ }^{5,6,7}$ Mannino et al. (2015), assessing the effect of adherence versus nonadherence to 2011 GOLD recommendations, observed that GOLD-adherent prescribing was associated with moderate reductions in the prevalence of COPD-related symptoms and the proportion of patients with all-cause hospitalizations and emergency department (ED) visits and respiratory-specific ED visits. ${ }^{8}$

As GOLD recommendations have evolved, there is a need to continue to evaluate their effect and quantify the effects of GOLD-compliant treatment from a clinical and economic perspective. The current study evaluated patients with COPD on maintenance therapy and their accordance with the 2017 GOLD treatment recommendations (current at the time of study execution) in a real-world U.S. commercially insured and Medicare Advantage population. The objective of this study was to compare COPD-related health care resource utilization (HRU), costs, and exacerbation rates among patients with COPD based on 2017 GOLD-compliant versus noncompliant status stratified by GOLD A/B or C/D (primary) and individual A/B/C/D (secondary) risk groups. ${ }^{2}$

\section{Methods}

\section{STUDY DESIGN AND DATA SOURCE}

This retrospective, observational cohort study compared individuals with COPD whose therapeutic regimens were compliant versus noncompliant with the 2017 GOLD treatment recommendations using the HealthCore Integrated Research Database (HIRD). ${ }^{2}$ The HIRD contains administrative medical and pharmacy claims data from enrollees in 14 commercial and Medicare Advantage health plans across the United States. The data are integrated across a broad spectrum of sources and types, including professional claims, facility claims, outpatient pharmacy claims, outpatient laboratory results, and enrollment information. The study received a Health Insurance Portability and Accountability Act waiver of authorization from an independent institutional review board.

\section{STUDY POPULATION}

After using a previously validated claims-based model to identify a COPD-prevalent population (Supplementary Table 1 , available in online article), ${ }^{9}$ the HIRD was queried to identify patients with $\geq 1$ COPD maintenance therapy (freeor fixed-dose formulations involving LAMA, LABA and/or ICS; Supplementary Table 2, available in online article) claim between January 1, 2014, and March 31, 2017, (intake period). The date of the earliest COPD maintenance treatment during this period was identified as the index date. Patients were required to be aged $\geq 40$ years at index, have $\geq 12$ months of 
pre-index (baseline) and $\geq 30$ days of post-index (follow-up) health plan enrollment, and no diagnosis for asthma, cystic fibrosis, and/or lung cancer any time during the study period (January 1, 2013-March 31, 2018).

\section{STUDY COHORTS AND EXPOSURE}

Patients were categorized into COPD ABCD risk groups according to the 2017 GOLD recommendations. ${ }^{2}$ We chose the most contemporary version in order to make our results most applicable to payers and clinicians in the current time period and have the highest utility.

Patients were classified into GOLD A/B or C/D risk groups if $0-1$ moderate and 0 severe exacerbations (A/B) $\mathrm{OR} \geq 2$ moderate and/or $\geq 1$ severe exacerbations (C/D) were observed during the baseline period. Moderate COPD exacerbations were defined based on the occurrence of an ED visit with a primary COPD diagnosis or $\geq 1$ claim for oral corticosteroid (OCS) or antibiotic therapy within 7 days of an office visit involving a COPD diagnosis in any position. A medical claim for an inpatient admission with a COPD diagnosis in the primary position constituted a severe exacerbation. All exacerbation events occurring $<30$ days apart were considered a single COPD exacerbation episode and assigned the highest observed severity.

GOLD classifies the $4 \mathrm{~A}, \mathrm{~B}, \mathrm{C}$, and D categories based on exacerbation history plus COPD symptom burden (high/ low). ${ }^{2}$ Highly symptomatic patients were identified from claims if they met $\geq 1$ of the following criteria during the baseline period: (a) $\geq 1$ medical claim for pulmonary rehabilitation; (b) $\geq 7$ pharmacy claims for a short-acting muscarinic antagonist (SAMA) and/or short-acting beta2agonist (SABA) over 12 months or $\geq 3$ pharmacy claims for a SAMA and/or a SABA over any 3-month period; (c) $\geq 3$ medical claims for an office visit with a COPD diagnosis over any 3-month period; (d) $\geq 3$ medical claims for an office visit with a dyspnea or cough diagnosis; (e) $\geq 1$ medical claim for an office visit with a dyspnea or cough diagnosis and $\geq 1$ medical claim for lung function testing or sputum culture; and/or (f) $\geq 1$ medical claim in the inpatient/ED setting for respiratory failure. Patients with COPD were identified as having low symptom burden if they did not meet any of these criteria. This exploratory algorithm was developed by sourcing expert clinical input and available published evidence in COPD and other respiratory conditions. ${ }^{10-13}$ This symptom algorithm in combination with the exacerbationbased classification placed patients into the individual GOLD A/B/C/D risk groups.

The combined A/B and C/D categorization was deemed as the primary analysis as those groupings were based on exacerbation history alone. The individual A/B/C/D category analysis was considered secondary since this classification also relied on a symptom burden algorithm that had not been previously utilized or validated.

The GOLD treatment recommendations provide guidance on COPD therapies for each of the ABCD risk groups (Supplementary Table 3, available in online article). For each patient, the maintenance regimens observed at index and during baseline were compared with the GOLD treatment guidance tailored to their individual $\mathrm{ABCD}$ risk group assignment. If the index maintenance medications and treatments before it were consistent with the GOLD treatment recommendations for their risk group, the patient was classified as compliant with GOLD recommendations; otherwise, the patient was classified as noncompliant. For example, if a patient with COPD categorized as GOLD B received either a LAMA or LABA monotherapy at index with no previous baseline maintenance medications or received an index LAMA+LABA with a baseline monoLAMA or LABA, then they would be considered as GOLD compliant. Use of any ICS-containing regimen would place them in the noncompliant strata, as GOLD does not list any ICS-containing regimens as recommended therapy for patients in the GOLD B category. For the primary analysis, where patients are categorized as A/B or C/D, the patients categorized as GOLD B in this example would be classified as compliant if they meet the compliance criteria for either GOLD A or B.

\section{OUTCOME MEASURES}

COPD-Related HRU and Costs. HRU and costs were considered as COPD-related if a medical claim carried a COPD and/or pneumonia diagnosis in any position or a medical/ pharmacy claim involved COPD maintenance or rescue therapies, OCS, or respiratory antibiotics (Supplementary Table 2, available in online article). HRU was stratified by an encounter's place of service (inpatient, ED, office visit, and other outpatient encounters) and reported per patient per year (PPPY). Costs were categorized by medical, pharmacy, and total, with the medical costs further grouped by the places of service as described above. In addition, costs reflected the amounts paid by both the plans and the patient (copay, deductible, and coinsurance) and reported on an annualized per-patient basis after inflating to 2017 U.S. dollars using the medical care component of the Consumer Price Index from the Bureau of Labor Statistics..$^{14}$ Unadjusted all-cause costs were also calculated and included all medical and pharmacy claims regardless of diagnosis.

COPD Exacerbation Risk. Risk for COPD exacerbations (severe and overall) was measured by calculating the (a) rate of exacerbations (expressed as number of exacerbations PPPY) and (b) time to first exacerbation during the 


\begin{tabular}{|c|c|c|c|c|c|c|c|}
\hline & \multicolumn{5}{|c|}{ Criteria } & \multicolumn{2}{|c|}{$\mathbf{n}$} \\
\hline 1 & \multicolumn{5}{|c|}{ Patients with COPD identified during the study period (January 1, 2013-March 31, 2018) } & \multicolumn{2}{|c|}{236,288} \\
\hline 2 & \multicolumn{5}{|c|}{$\begin{array}{l}\text { Patients with COPD with } \geq 1 \text { claim for a COPD maintenance treatment during the intake } \\
\text { period (January 1, 2014-March 31, 2017) }\end{array}$} & \multicolumn{2}{|c|}{123,301} \\
\hline 3 & \multicolumn{5}{|c|}{ Patients with COPD aged $\geq 40$ years } & \multicolumn{2}{|c|}{121,828} \\
\hline 4 & \multicolumn{5}{|c|}{$\begin{array}{l}\text { Patients with COPD with no claims with a diagnosis for asthma, cystic fibrosis, or lung } \\
\text { cancer during the study period }\end{array}$} & \multicolumn{2}{|c|}{68,446} \\
\hline 5 & \multicolumn{5}{|c|}{$\begin{array}{l}\text { Patients with COPD with } \geq 12 \text {-months pre-index continuous medical and pharmacy } \\
\text { eligibility }\end{array}$} & \multicolumn{2}{|c|}{45,298} \\
\hline 6 & \multicolumn{5}{|c|}{$\begin{array}{l}\text { Patients with COPD with } \geq 30 \text { days post-index continuous medical and pharmacy } \\
\text { eligibility }\end{array}$} & \multicolumn{2}{|c|}{44,917} \\
\hline \multicolumn{4}{|c|}{$\begin{array}{c}\text { GOLD A/B } \\
\mathbf{n}(\%) \\
38,382(85.45)\end{array}$} & \multicolumn{4}{|c|}{$\begin{array}{c}\text { GOLD C/D } \\
\mathbf{n}(\%) \\
6,535(14.55)\end{array}$} \\
\hline \multicolumn{2}{|c|}{$\begin{array}{c}\text { Compliant } \\
12,631(32.91)\end{array}$} & \multicolumn{2}{|c|}{$\begin{array}{l}\text { Noncompliant } \\
25,751(67.09)\end{array}$} & \multicolumn{2}{|c|}{$\begin{array}{c}\text { Compliant } \\
3,852(58.94)\end{array}$} & \multicolumn{2}{|c|}{$\begin{array}{l}\text { Noncompliant } \\
2,683(41.06)\end{array}$} \\
\hline \multicolumn{2}{|c|}{$\begin{array}{c}\text { GOLD A } \\
19,345(43.07)\end{array}$} & \multicolumn{2}{|c|}{$\begin{array}{c}\text { GOLD B } \\
19,037(42.38)\end{array}$} & \multicolumn{2}{|c|}{$\begin{array}{c}\text { GOLD C } \\
1,865(4.15)\end{array}$} & \multicolumn{2}{|c|}{$\begin{array}{c}\text { GOLD D } \\
4,670(10.40)\end{array}$} \\
\hline $\begin{array}{c}\text { Compliant } \\
6,488 \\
(33.54)\end{array}$ & $\begin{array}{c}\text { Noncompliant } \\
12,857 \\
(66.46)\end{array}$ & $\begin{array}{c}\text { Compliant } \\
4,888 \\
(25.68) \\
\end{array}$ & $\begin{array}{c}\text { Noncompliant } \\
14,149 \\
(74.32)\end{array}$ & $\begin{array}{c}\text { Compliant } \\
627 \\
(33.62) \\
\end{array}$ & $\begin{array}{c}\text { Noncompliant } \\
1,238 \\
(66.38)\end{array}$ & $\begin{array}{c}\text { Compliant } \\
2,871 \\
(61.48) \\
\end{array}$ & $\begin{array}{c}\text { Noncompliant } \\
1,799 \\
(38.52) \\
\end{array}$ \\
\hline
\end{tabular}

Note: "Compliant" was defined as receipt of COPD maintenance therapy that was consistent with the 2017 GOLD recommendations. $C O P D=$ chronic obstructive pulmonary disease $G O L D=$ Global Initiative for Chronic Obstructive Lung Disease.

post-index period. Overall exacerbations included either moderate or severe exacerbations (definitions described previously). Severe exacerbations were only analyzed in the primary risk group categorization of GOLD A/B and C/D.

\section{STATISTICAL ANALYSIS}

Multivariable models were constructed to assess the effect of compliance (yes/no) to GOLD treatment recommendations on COPD-related HRU and costs, exacerbation rates, and time to first exacerbation per the primary (A/B and $\mathrm{C} / \mathrm{D})$ and secondary $(\mathrm{A} / \mathrm{B} / \mathrm{C} / \mathrm{D})$ risk group categorization. Comparisons of study endpoints between the compliant versus noncompliant groups were conducted using methodologies appropriate to the distribution of the measure and adjusting for each patient's available follow-up. A generalized linear model (GLM) with negative binomial distribution and log link was used to assess COPD-related HRU, a GLM with gamma distribution and log link was used to assess COPDrelated costs, a negative binomial regression was used to assess exacerbation rates, and a Cox proportional hazards regression was used to assess time to first exacerbation.

For each model, specific covariates included were determined based upon clinical rationale and descriptive results and selected by the backward selection method with an exit threshold of $\mathrm{P} \geq 0.05$ (Supplementary Table 4, available in online article). The following covariates were initially included for model selection in all the models: age at index, gender, region of residence, insurance type, Medicare Advantage enrollment, treating provider specialty on index date, index year, Elixhauser comorbidity index, baseline comorbidities, COPD exacerbation count, exacerbation costs, and COPD-related total, medical, and pharmacy costs. In addition, unadjusted all-cause costs were calculated for the GOLD A/B and C/D risk groups by regimen compliance status. A conventional alpha of 0.05 and 2-tailed level of significance were used for all outcomes. All analyses were performed using SAS Enterprise 7.1 (SAS Institute, Cary, NC).

\section{Results}

\section{SAMPLE IDENTIFICATION AND CHARACTERISTICS}

A total of 236,288 patients with COPD were identified in the HIRD between January 1, 2013, and March 31, 2018 (Table 1). Among these, 123,301 patients (52.2\%) received a COPD maintenance treatment between January 1, 2014, and March 31, 2017. After applying all study entry criteria, a 


\section{TABLE 2 Baseline Demographic and Clinical Characteristics, COPD-Related Health Care Resource Utilization, and Costs ${ }^{\mathrm{a}}$}

\begin{tabular}{|c|c|c|c|c|c|c|c|c|}
\hline \multirow{3}{*}{$\frac{\text { Characteristics }}{\text { Age, years, mean (SD) }}$} & \multicolumn{4}{|c|}{ GOLD A/B } & \multicolumn{4}{|c|}{ GOLD C/D } \\
\hline & \multicolumn{2}{|c|}{$\begin{array}{c}\text { Compliant } \\
n=12,631\end{array}$} & \multicolumn{2}{|c|}{$\begin{array}{c}\text { Noncompliant } \\
n=25,751\end{array}$} & \multicolumn{2}{|c|}{$\begin{array}{c}\text { Compliant } \\
n=3,852\end{array}$} & \multicolumn{2}{|c|}{$\begin{array}{c}\text { Noncompliant } \\
n=2,683\end{array}$} \\
\hline & 69.32 & $(10.92)^{b}$ & 68.48 & $(11.45)$ & 69.35 & $(10.38)$ & 69.25 & $(11.08)$ \\
\hline Gender, female, n (\%) & 6,222 & $(49.26)^{b}$ & 12,161 & $(47.23)$ & 2,013 & $(52.26)$ & 1,406 & $(52.40)$ \\
\hline \multicolumn{9}{|l|}{ Region of residence, $n(\%)^{b}$} \\
\hline Northeast & 2,307 & $(18.26)$ & 4,093 & $(15.89)$ & 678 & $(17.60)$ & 362 & $(13.49)$ \\
\hline Midwest & 4,869 & $(38.55)$ & 10,467 & $(40.65)$ & 1,589 & $(41.25)$ & 1,296 & $(48.30)$ \\
\hline South & 3,804 & $(30.12)$ & 7,838 & $(30.44)$ & 1,152 & $(29.91)$ & 823 & $(30.67)$ \\
\hline West & 1,651 & $(13.07)$ & 3,353 & $(13.02)$ & 433 & $(11.21)$ & 202 & (7.53) \\
\hline Medicare Advantage, n (\%) & 4,150 & $(32.86)^{b}$ & 8,174 & $(31.74)$ & 1,566 & $(40.65)$ & 1,121 & $(41.78)$ \\
\hline \multicolumn{9}{|l|}{ Prescriber specialty on index date, $n(\%)^{b}$} \\
\hline Pulmonologist & 3,467 & $(27.45)$ & 5,562 & $(21.60)$ & 1,250 & $(32.45)$ & 524 & $(19.53)$ \\
\hline Primary care $^{c}$ & 6,353 & $(50.30)$ & 12,833 & $(49.83)$ & 1,787 & $(46.39)$ & 1,387 & $(51.70)$ \\
\hline Others $^{\mathrm{d}}$ & 2,617 & $(20.72)$ & 6,629 & $(25.74)$ & 780 & $(20.25)$ & 717 & $(26.72)$ \\
\hline Unknown & 194 & $(1.54)$ & 727 & $(2.82)$ & 35 & $(0.91)$ & 55 & $(2.05)$ \\
\hline Elixhauser comorbidity index, mean (SD) & 4.24 & $(2.84)$ & 4.26 & $(2.94)$ & 5.00 & $(2.95)^{b}$ & 5.50 & $(3.21)$ \\
\hline \multicolumn{9}{|l|}{ Specific comorbidities, n (\%) } \\
\hline Anxiety & 1,854 & $(14.68)^{\mathrm{b}}$ & 4,029 & $(15.65)$ & 918 & $(23.83)$ & 671 & $(25.01)$ \\
\hline Atrial fibrillation & 1,930 & $(15.28)$ & 3,820 & $(14.83)$ & 666 & $(17.29)$ & 514 & $(19.16)$ \\
\hline Depression & 2,217 & $(17.55)$ & 4,650 & $(18.06)$ & 900 & $(23.36)^{b}$ & 677 & $(25.23)$ \\
\hline Diabetes mellitus & 3,151 & $(24.95)^{\mathrm{b}}$ & 6,846 & $(26.59)$ & 996 & $(25.86)^{b}$ & 836 & $(31.16)$ \\
\hline Dyspnea & 6,227 & $(49.30)$ & 12,806 & $(49.73)$ & 2,873 & $(74.58)^{\mathrm{b}}$ & 2,115 & $(78.83)$ \\
\hline Congestive heart failure & 2,275 & $(18.01)$ & 4,821 & $(18.72)$ & 994 & $(25.80)^{b}$ & 850 & $(31.68)$ \\
\hline Myocardial infarction & 929 & $(7.35)$ & 1,911 & $(7.42)$ & 384 & $(9.97)^{\mathrm{b}}$ & 325 & $(12.11)$ \\
\hline Hypertension & 8,976 & $(71.06)$ & 18,220 & $(70.75)$ & 2,885 & $(74.90)^{\mathrm{b}}$ & 2,075 & $(77.34)$ \\
\hline Pneumonia & 1,657 & $(13.12)^{b}$ & 4,023 & $(15.62)$ & 1,307 & $(33.93)^{b}$ & 991 & $(36.94)$ \\
\hline Pulmonary fibrosis & 561 & $(4.44)$ & 1,107 & $(4.30)$ & 303 & $(7.87)$ & 201 & (7.49) \\
\hline Renal disease & 1,515 & (11.99) & 3,188 & $(12.38)$ & 497 & $(12.90)^{b}$ & 425 & $(15.84)$ \\
\hline Obstructive sleep apnea & 1,582 & $(12.52)$ & 3,081 & $(11.96)$ & 613 & $(15.91)$ & 387 & $(14.42)$ \\
\hline \multicolumn{9}{|l|}{ Exacerbations, n (\%) } \\
\hline Any exacerbations & 2,657 & $(21.04)^{b}$ & 5,958 & $(23.14)$ & 3,852 & $(100.0)^{b}$ & 2,683 & $(100.0)$ \\
\hline Severe exacerbations & 0 & $(0.0)$ & 0 & $(0.0)$ & 1,497 & $(38.86)^{\mathrm{b}}$ & 1,415 & $(52.74)$ \\
\hline Moderate exacerbations & 2,657 & $(21.04)^{b}$ & 5,958 & $(23.14)$ & 2,987 & $(77.54)^{\mathrm{b}}$ & 1,652 & $(61.57)$ \\
\hline \multicolumn{9}{|c|}{ Cost per exacerbation episode per patient, mean (SD), \$ } \\
\hline Any exacerbations & 72 & $(419)$ & 87 & $(483)^{\mathrm{b}}$ & 4,124 & $(10,244)^{b}$ & 5,353 & $(13,542)$ \\
\hline Severe exacerbations & 0 & $(0)$ & 0 & $(0)$ & 5,141 & $(12,213)^{b}$ & 6,221 & $(16,982)$ \\
\hline Moderate exacerbations & 72 & $(419)$ & 87 & $(483)^{\mathrm{b}}$ & 229 & $(543)^{b}$ & 206 & $(480)$ \\
\hline
\end{tabular}




\section{TABLE 2 Baseline Demographic and Clinical Characteristics, COPD-Related Health Care Resource Utilization, and Costs $^{\mathrm{a}}$ (continued)}

\begin{tabular}{|c|c|c|c|c|c|c|c|c|}
\hline \multirow[b]{2}{*}{ Characteristics } & \multicolumn{4}{|c|}{ GOLD A/B } & \multicolumn{4}{|c|}{ GOLD C/D } \\
\hline & \multicolumn{2}{|c|}{$\begin{array}{l}\text { Compliant } \\
n=12,631\end{array}$} & \multicolumn{2}{|c|}{$\begin{array}{c}\text { Noncompliant } \\
n=25,751\end{array}$} & \multicolumn{2}{|c|}{$\begin{array}{c}\text { Compliant } \\
\mathrm{n}=3,852\end{array}$} & \multicolumn{2}{|c|}{$\begin{array}{c}\text { Noncompliant } \\
n=2,683\end{array}$} \\
\hline \multicolumn{9}{|c|}{ COPD-related HRU, number of events per patient-year, mean (SD) } \\
\hline Inpatient admissions & 0.27 & $(0.67)^{\mathrm{b}}$ & 0.30 & $(0.70)$ & 0.88 & $(1.22)^{\mathrm{b}}$ & 1.04 & $(1.24)$ \\
\hline ED & 0.14 & $(0.47)$ & 0.15 & $(0.47)$ & 0.39 & $(0.87)^{\mathrm{b}}$ & 0.43 & $(0.96)$ \\
\hline Office visits & 2.09 & $(2.39)^{\mathrm{b}}$ & 1.91 & $(2.21)$ & 5.78 & $(4.04)^{\mathrm{b}}$ & 4.20 & $(3.81)$ \\
\hline Other outpatient encounters & 5.2 & $(10.82)$ & 5.2 & $(10.66)$ & 14.52 & $(20.04)^{b}$ & 11.47 & $(19.44)$ \\
\hline \multicolumn{9}{|l|}{ COPD-related costs, mean (SD), \$ } \\
\hline Medical & 8,615 & $(31,450)^{b}$ & 9,035 & $(33,219)$ & 17,903 & $(32,282)$ & 18,485 & $(37,906)$ \\
\hline Pharmacy & 1,481 & $(1,988)^{\mathrm{b}}$ & 1,112 & $(1,888)$ & 3,015 & $(2,814)^{\mathrm{b}}$ & 641 & $(1,383)$ \\
\hline Total & 10,097 & $(31,445)^{b}$ & 10,147 & $(33,198)$ & 20,918 & $(32,329)^{b}$ & 19,126 & $(37,903)$ \\
\hline \multirow{2}{*}{ Length of follow-up, years, mean (SD) Median } & 0.94 & $(0.18)^{b}$ & 0.93 & $(0.19)$ & 0.91 & $(0.21)^{\mathrm{b}}$ & 0.88 & $(0.24)$ \\
\hline & \multicolumn{2}{|c|}{1.00} & \multicolumn{2}{|c|}{1.00} & \multicolumn{2}{|c|}{1.00} & \multicolumn{2}{|c|}{1.00} \\
\hline
\end{tabular}

${ }^{a}$ Costs are adjusted to 2017 dollars as per the most recent medical care price index information provided by the Bureau of Labor Statistics.

bSignificantly different with $P$ value $<0.05$ vs. corresponding noncompliant group.

'Primary care includes family medicine, general practitioners, and internal medicine.

${ }^{\mathrm{d} O}$ Others includes nonphysician prescribers such as nurse practitioners and physician assistants.

$C O P D=$ chronic obstructive pulmonary disease; $E D=$ emergency department; GOLD=Global Initiative for Chronic Obstructive Lung Disease; HRU = health care resource utilization; $S D=$ standard deviation.

total of 44,917 patients were included in the final analytical sample.

A majority of the patients with COPD were categorized into GOLD A/B (85.5\%), while $14.5 \%$ were categorized into GOLD C/D. For individual GOLD risk groups, $43.1 \%$ of patients were GOLD A, 42.4\% GOLD B, 4.1\% GOLD C, and 10.4\% GOLD D. In terms of 2017 GOLD treatment recommendation compliance, only $32.9 \%$ of patients categorized in the GOLD A/B group met the criteria while $58.9 \%$ were categorized as compliant in the GOLD C/D group. Compliance for each of the individual GOLD risk groups was observed in $33.5 \%, 25.7 \%, 33.6 \%$, and $61.5 \%$ of patients in GOLD A, B, C, and D, respectively.

As for the noncompliant treatment regimens observed among patients in the GOLD A/B group, 90.4\% received some form of ICS-containing therapies, with overuse of LABA/ICS found in $68.8 \%$ of these patients and open triple (LAMA/LABA/ICS) combinations in 9.3\%. The remaining $12.4 \%$ involved other ICS-containing regimens. Among the patients in the GOLD C/D group who were noncompliant with GOLD, more than half in the noncompliant group (56.8\%) received LABA/ICS but without a previous LAMAcontaining monotherapy.
Table 2 presents baseline demographic and clinical characteristics, exacerbations, and COPD-related HRU and costs for the GOLD A/B and C/D groups. The cohorts were evenly split regarding gender, with a mean age of approximately 69 years. In approximately $50 \%$ of patients, the index maintenance medication was prescribed by a primary care physician. While the median follow-up times were similar across the groups, their means were slightly longer $(P<0.0001)$ for the compliant groups.

\section{COPD-RELATED HRU}

Results of the multivariate analysis estimating the effect of complying with GOLD recommendations versus noncompliance in terms of mean difference in visits PPPY by place of service are listed in Table 3. A negative result in the difference column implies lower use within the GOLDcompliant subgroup.

For those in the GOLD A/B group, compliance to GOLD recommendations resulted in 0.02 fewer hospitalizations PPPY versus the noncompliant regimen $(P=0.040)$. We also found a significantly greater number of office visits among patients who were compliant, with 0.06 more visits PPPY (or $6+$ visits per 100 patient-years; $P=0.018$ ). Within the individual GOLD A group, none of the differences in 


\section{TABLE 3}

\begin{tabular}{|c|c|c|c|c|}
\hline & Compliant & Noncompliant & Mean Difference $^{\mathrm{b}}(95 \% \mathrm{CI})$ & P Value \\
\hline \multicolumn{5}{|l|}{ Inpatient } \\
\hline GOLD A/B & 0.31 & 0.33 & $-0.02 \quad(-0.03,0.00)$ & 0.040 \\
\hline GOLD C/D & 0.65 & 0.77 & $-0.12 \quad(-0.17,-0.07)$ & $<0.0001$ \\
\hline GOLD A & 0.26 & 0.27 & $-0.01 \quad(-0.03,0.01)$ & 0.374 \\
\hline GOLD B & 0.38 & 0.39 & $-0.01 \quad(-0.03,0.01)$ & 0.408 \\
\hline GOLD C & 0.47 & 0.54 & $-0.07 \quad(-0.14,0.01)$ & 0.085 \\
\hline GOLD D & 0.72 & 0.86 & $-0.14(-0.21,-0.08)$ & $<0.0001$ \\
\hline
\end{tabular}

Emergency department

\begin{tabular}{|c|c|c|c|c|}
\hline GOLD A/B & 0.16 & 0.17 & $0.00 \quad(-0.01,0.01)$ & 0.589 \\
\hline GOLD C/D & 0.33 & 0.38 & $-0.06 \quad(-0.09,-0.02)$ & 0.003 \\
\hline GOLD A & 0.15 & 0.15 & $-0.004(-0.02,0.01)$ & 0.604 \\
\hline GOLD B & 0.18 & 0.19 & $-0.011(-0.03,0.00)$ & 0.165 \\
\hline GOLD C & 0.24 & 0.33 & $-0.08 \quad(-0.13,-0.02)$ & 0.017 \\
\hline GOLD D & 0.36 & 0.42 & $-0.06 \quad(-0.10,-0.01)$ & 0.022 \\
\hline \multicolumn{5}{|l|}{ Office visits } \\
\hline GOLD A/B & 2.97 & 2.90 & $0.06 \quad(0.01,0.12)$ & 0.018 \\
\hline GOLD C/D & 4.60 & 4.88 & $-0.28 \quad(-0.45,-0.10)$ & 0.002 \\
\hline GOLD A & 2.73 & 2.67 & $0.06 \quad(-0.01,0.13)$ & 0.112 \\
\hline GOLD B & 3.34 & 3.22 & $0.11 \quad(0.03,0.21)$ & 0.011 \\
\hline GOLD C & 3.73 & 4.12 & $-0.39 \quad(-0.66,-0.11)$ & 0.008 \\
\hline GOLD D & 5.04 & 5.20 & $-0.16 \quad(-0.38,0.08)$ & 0.184 \\
\hline \multicolumn{5}{|c|}{ Other outpatient visits } \\
\hline GOLD A/B & 6.27 & 6.42 & $-0.15 \quad(-0.32,0.03)$ & 0.100 \\
\hline GOLD C/D & 12.87 & 13.67 & $-0.80 \quad(-1.46,-0.09)$ & 0.027 \\
\hline GOLD A & 4.99 & 4.86 & $0.12 \quad(-0.07,0.33)$ & 0.219 \\
\hline GOLD B & 7.75 & 8.47 & $-0.72 \quad(-1.02,-0.40)$ & $<0.0001$ \\
\hline GOLD C & 8.17 & 9.48 & $-1.31 \quad(-2.22,-0.29)$ & 0.013 \\
\hline GOLD D & 15.13 & 16.56 & $-1.43 \quad(-2.32,-0.49)$ & 0.004 \\
\hline
\end{tabular}

aOther medical (e.g., skilled nursing facility) health care encounters were not modeled.

${ }^{b}$ Mean differences are from negative binomial models used for count variables. All models are offset by length of follow-up. Statistical comparisons are comparing compliant to GOLD with noncompliant to GOLD (reference group). Model covariates include demographics, baseline comorbidities, baseline exacerbation outcomes, baseline health care utilization, and costs, which were selected by backward selection method with an exit threshold of $P \geq 0.05$.

$\mathrm{Cl}=$ confidence interval $\mathrm{COPD}=$ chronic obstructive pulmonary disease $; \mathrm{GOL}=\mathrm{Global}$ Initiative for Chronic Obstructive Lung Disease.

HRU between the compliance status groups were statistically significant. For the GOLD B group, office visits were significantly greater $(0.11$
PPPY; P=0.011), but other outpatient encounters were significantly less $(-0.72$ PPPY; $\mathrm{P}<0.001)$ in the compliant subgroup.
For the GOLD C/D group, we saw statistically significant lower HRU in the GOLD-compliant versus noncompliant groups across the board. GOLD compliance was associated with 12 fewer hospitalizations, 6 fewer ED visits, 28 fewer office visits and 80 fewer outpatient visits per 100 patient-years (all $\mathrm{P}<0.05$ ). Within the individual GOLD $C$ group, all except the inpatient encounters occurred significantly less frequently in the patients on a GOLD-compliant regimen (all $\mathrm{P}<0.05$ ). For the individual GOLD D group, all but office visits occurred significantly less frequently in the patients on a GOLD-compliant regimen (all $\mathrm{P}<0.05$ ).

Unadjusted follow-up all-cause and COPD-related HRU are displayed in Supplementary Table 5a (available in online article).

\section{COPD-RELATED COSTS}

Adjusted mean cost differences between patients on compliant versus noncompliant regimens are shown in Table 4. Similar to the HRU results, a negative result implies lower cost within the GOLD-compliant subgroup.

In the GOLD A/B group, medical costs were significantly lower in patients on a GOLD-compliant regimen (-\$706; $P=0.002)$, while pharmacy costs were significantly higher $(\$ 440 ; \mathrm{P}<0.001)$. This resulted in a total burden that was lower overall (-\$265) but not statistically significant $(\mathrm{P}=0.175)$. The majority of the medical cost reduction was due to the decreased inpatient costs observed (-\$802; $P=0.003)$. These differences were driven by the individual GOLD B group, in which significantly lower spending was observed for medical services $(-\$ 1,534 ; P<0.001)$ in turn driven by the inpatient setting $(-\$ 1,318$; $\mathrm{P}=0.006)$, pharmacy $(-\$ 335 ; \mathrm{P}<0.001)$, and overall $(-\$ 1,869 ; \quad P<0.001)$ in the GOLD-compliant subgroup. For the GOLD A group, compliance 
TABLE 4 Adjusted Mean Difference in COPD-Related Costs in Patients with COPD on Compliant vs. Noncompliant GOLD Treatment Regimens (per patient per year) ${ }^{a}$

\begin{tabular}{|c|c|c|c|c|}
\hline & Compliant & Noncompliant & Mean Difference ${ }^{b}(95 \% \mathrm{CI})$ & $P$ Value \\
\hline \multicolumn{5}{|c|}{ Total COPD medical costs, $\$$} \\
\hline GOLD A/B & 11,061 & 11,767 & $-706(-1,127,-268)$ & 0.002 \\
\hline GOLD C/D & 19,089 & 21,313 & $-2,224 \quad(-3,643,-690)$ & 0.005 \\
\hline GOLD A & 9,485 & 9,383 & $103(-414,650)$ & 0.703 \\
\hline GOLD B & 12,597 & 14,131 & $-1,534 \quad(-2,233,-794)$ & $<0.0001$ \\
\hline GOLD C & 12,469 & 15,172 & $-2,703 \quad(-4,485,-624)$ & 0.013 \\
\hline GOLD D & 21,273 & 24,140 & $-2,868 \quad(-4,709,-851)$ & 0.006 \\
\hline \multicolumn{5}{|l|}{ Inpatient, \$ } \\
\hline GOLD A/B & 8,111 & 8,913 & $-802(-1,288,-285)$ & 0.003 \\
\hline GOLD C/D & 14,377 & 16,497 & $-2,120 \quad(-3,910,-75)$ & 0.043 \\
\hline GOLD A & 6,910 & 7,057 & $-147 \quad(-725,484)$ & 0.637 \\
\hline GOLD B & 9,376 & 10,695 & $-1,318 \quad(-2,155,-400)$ & 0.006 \\
\hline GOLD C & 9,601 & 11,615 & $-2,014 \quad(-4,214,839)$ & 0.151 \\
\hline GOLD D & 16,026 & 18,635 & $-2,609 \quad(-4,953,137)$ & 0.062 \\
\hline \multicolumn{5}{|c|}{ Emergency department, \$ } \\
\hline GOLD A/B & 355 & 379 & $-24 \quad(-43,-5)$ & 0.016 \\
\hline GOLD C/D & 712 & 806 & $-94(-177,-1)$ & 0.049 \\
\hline GOLD A & 320 & 331 & $-10 \quad(-33,15)$ & 0.414 \\
\hline GOLD B & 360 & 437 & $-76 \quad(-105,-46)$ & $<0.0001$ \\
\hline GOLD C & 540 & 591 & $-51 \quad(-167,95)$ & 0.457 \\
\hline GOLD D & 775 & 902 & $-127 \quad(-233,-4)$ & 0.044 \\
\hline \multicolumn{5}{|l|}{ Office, $\$$} \\
\hline GOLD A/B & $\$ 327$ & 313 & $14(5,23)$ & 0.002 \\
\hline GOLD C/D & $\$ 512$ & 470 & $42(15,69)$ & 0.002 \\
\hline GOLD A & $\$ 296$ & 284 & $13(1,25)$ & 0.027 \\
\hline GOLD B & $\$ 349$ & 349 & $1(-13,15)$ & 0.938 \\
\hline GOLD C & $\$ 385$ & 410 & $-25 \quad(-63,16)$ & 0.218 \\
\hline GOLD D & $\$ 552$ & 504 & $-48 \quad(14,84)$ & 0.005 \\
\hline \multicolumn{5}{|c|}{ Other outpatient, \$ } \\
\hline GOLD A/B & 2,057 & 1,925 & $131(49,218)$ & 0.002 \\
\hline GOLD C/D & 3,168 & 3,092 & $76(-168,341)$ & 0.552 \\
\hline GOLD A & 1,783 & 1,511 & $272 \quad(-169,382)$ & $<0.0001$ \\
\hline GOLD B & 2,274 & 2,377 & $-103 \quad(-234,37)$ & 0.147 \\
\hline GOLD C & 1,714 & 2,187 & $-473(-738,-160)$ & 0.004 \\
\hline GOLD D & 3,605 & 3,582 & $23(-292,368)$ & 0.891 \\
\hline
\end{tabular}

continued on next page was associated with significantly increased costs for other outpatient encounters (\$272; $\mathrm{P}<0.001)$, pharmacy (\$599; $\mathrm{P}<0.001)$, and total spend ( $\$ 692$; $\mathrm{P}=0.003)$.

Directionally similar results were observed in the GOLD C/D group, where medical costs were significantly lower in patients on a GOLD-compliant regimen $(-\$ 2,224 ; \quad P=0.005)$, while pharmacy costs were significantly higher $(\$ 1,753 ; \mathrm{P}<0.001)$. As with the GOLD A/B group, this resulted in a total cost that was lower overall $(-\$ 471)$ but not statistically significant $(P=0.504)$. The majority of the lower total medical cost was associated with decreased inpatient costs $(-\$ 2,120$; $\mathrm{P}=0.043)$. Within the individual GOLD $\mathrm{C}$ and $\mathrm{D}$ groups, medical costs were significantly lower in the compliant group $(-\$ 2,703 ; P=0.013$ and $-\$ 2,868$; $P=0.006$, respectively), driven by lower inpatient costs $(-\$ 2,014 ; P=0.151$ and $-\$ 2,609 ; P=0.062$, respectively) for those patients on a GOLD-compliant regimen. Pharmacy costs were significantly lower in the GOLD C group $(-\$ 340 ; \quad P=0.005)$ and significantly higher in the GOLD D group $(\$ 1,824$; $\mathrm{P}<0.001)$ when compliant. Total costs were lower in the GOLD C $(-\$ 3,043$; $P=0.002)$ and GOLD D groups $(-\$ 1,043$; $\mathrm{P}=0.261)$ on a GOLD-compliant regimen, but were statistically significant only in the GOLD C group.

Unadjusted all-cause and COPDrelated costs are displayed in Supplementary Table 5b (available in online article).

\section{EXACERBATION RISK}

In the GOLD A/B group, the overall exacerbation rate was lower in the compliant versus noncompliant group (0.54 vs. 0.59 , rate ratio $[R R]=0.93$; 95\% CI=0.89-0.96; $P<0.001$; Figure 1). The severe COPD exacerbation rate, while slightly lower in the compliant group, was not statistically significant 


\section{TABLE 4}

Adjusted Mean Difference in COPD-Related Costs in Patients with COPD on Compliant vs. Noncompliant GOLD Treatment Regimens (per patient per year) ${ }^{a}$ (continued)

\begin{tabular}{|c|c|c|c|c|}
\hline & Compliant & Noncompliant & Mean Difference $^{b}(95 \% \mathrm{CI})$ & P Value \\
\hline \multicolumn{5}{|c|}{ COPD pharmacy costs, \$ } \\
\hline GOLD A/B & 2,983 & 2,542 & $440(378,504)$ & $<0.0001$ \\
\hline GOLD C/D & 4,105 & 2,353 & $1,753 \quad(1,562,1,952)$ & $<0.0001$ \\
\hline GOLD A & 2,892 & 2,303 & $589(508,672)$ & $<0.0001$ \\
\hline GOLD B & 2,634 & 2,969 & $-335(-422,-245)$ & $<0.0001$ \\
\hline GOLD C & 2,553 & 2,893 & $-340(-553,-107)$ & 0.005 \\
\hline GOLD D & 4,343 & 2,519 & $1,824 \quad(1,578,2,085)$ & $<0.0001$ \\
\hline \multicolumn{5}{|c|}{ Total COPD costs, \$ } \\
\hline GOLD A/B & 14,043 & 14,309 & $-265(-640,119)$ & 0.175 \\
\hline GOLD C/D & 23,194 & 23,665 & $-471 \quad(-1,801,940)$ & 0.504 \\
\hline GOLD A & 12,377 & 11,685 & $692(-231,1,171)$ & 0.003 \\
\hline GOLD B & 15,231 & 17,100 & $-1,869 \quad(-2,477,-1,236)$ & $<0.0001$ \\
\hline GOLD C & 15,022 & 18,065 & $-3,043 \quad(-4,670,-1,218)$ & 0.002 \\
\hline GOLD D & 25,616 & 26,659 & $-1,043 \quad(-2,767,805)$ & 0.261 \\
\hline
\end{tabular}

${ }^{a}$ Costs for other medical (e.g., skilled nursing facility) were not modeled.

${ }^{b}$ Mean differences are from gamma regression model (link $\left.=l o g\right)$. All models are adjusted for length of follow-up. Statistical comparisons are comparing compliant to GOLD with noncompliant to GOLD (reference group). Model covariates include demographics, baseline comorbidities, baseline exacerbation outcomes, baseline health care utilization, and costs, which were selected by backward selection method with an exit threshold of $P \geq 0.05$.

$\mathrm{Cl}=$ confidence interval; $\mathrm{COPD}=$ chronic obstructive pulmonary disease; $\mathrm{GOLD}=\mathrm{Global}$ Initiative for Chronic Obstructive Lung Disease.

(0.11 vs. $0.12, \mathrm{RR}=0.91 ; 95 \% \mathrm{CI}=0.82-$ $1.02 ; \mathrm{P}=0.118)$. In the GOLD C/D group, the compliant group had a lower rate for both overall $(1.20$ vs. 1.27 , $\mathrm{RR}=0.94 ; 95 \% \mathrm{CI}=0.90-0.99 ; \mathrm{P}=0.028)$ and severe $(0.15$ vs. $0.18, R R=0.83$; 95\% $\mathrm{CI}=0.71-0.96 ; \mathrm{P}=0.010)$ exacerbation outcomes versus those found in the noncompliant group.

Results in the individual GOLD groups were similar to that in the primary combination groups. In the GOLD A group, compliance status was associated with a significantly lower overall COPD exacerbation rate $(0.41$ vs. $0.46, \mathrm{RR}=0.90 ; 95 \% \mathrm{CI}=0.85-0.95$; $\mathrm{P}<0.001)$. For the other risk groups, although exacerbation rates were numerically lower in the compliant versus noncompliant group, these differences were not statistically significant (Figure 1).

In the GOLD A/B group, the time to first exacerbation was longer among the compliant versus noncompliant group (137 vs. 129 days, respectively) and was associated with a significantly reduced risk of overall exacerbations (hazard ratio $[\mathrm{HR}]=0.92 ; 95 \% \mathrm{CI}=0.88-$ $0.95 ; \mathrm{P}<0.001)$. The risk of severe exacerbations was not significantly reduced in this group $(\mathrm{HR}=0.94$; $95 \% \mathrm{CI}=0.85-1.05 ; \mathrm{P}=0.297)$. In the GOLD C/D group, the time to first COPD exacerbation was longer in the compliant group compared with the noncompliant group (112 vs. 97 days, respectively) and was associated with significantly reduced risk for both overall exacerbations $(\mathrm{HR}=0.88$;
95\% $\quad \mathrm{CI}=0.82-0.95 ; \quad \mathrm{P}<0.001) \quad$ and severe exacerbations $\quad(\mathrm{HR}=0.81$; 95\% CI $=0.69-0.95 ; P=0.01$ ).

Time to first exacerbation and risk of overall exacerbations in the individual GOLD ABCD groups was similar to the primary analyses above. All the individual GOLD ABCD-compliant risk groups were associated with statistically significant risk reductions in overall exacerbations compared with the noncompliant risk groups ([GOLD A: HR $=0.89 ; 95 \% \mathrm{CI}=0.85-$ 0.94; $\mathrm{P}<0.001]$; [GOLD B: $\mathrm{HR}=0.93$; 95\% CI $=0.88-0.99 ; P=0.017] ;$ [GOLD C: $\mathrm{HR}=0.87 ; 95 \% \mathrm{CI}=0.76-0.99 ; \mathrm{P}=0.029]$; [GOLD D: $H R=0.90 ; 95 \% \mathrm{CI}=0.83-$ $0.98 ; P=0.013])$.

\section{Discussion}

Guideline recommendations and consensus statements such as those described in the GOLD report are developed based on the latest scientific evidence and expert guidance to provide evidence-based treatment recommendations and, ultimately, improve patient outcomes. However, if these recommendations are not followed, optimizing outcomes will be difficult, resulting in negative consequences for patients and to the health care system overall.

Almost half (48\%) of patients with COPD in our population did not receive a COPD maintenance medication. While this is appropriate for some patients within the low-risk GOLD A group, the magnitude of the estimate suggests the underprescribing of maintenance drugs. Previous studies found similar or greater percentages than what this study observed., ${ }^{4,15}$ Make et al. (2012) analyzed more than 50,000 patients and reported that $66 \%$ of commercially insured and $71 \%$ of Medicare patients did not have a prescription claim for a COPD maintenance therapy. ${ }^{4}$ Similarly, Diette et al. (2015) reported that $55 \%$ of patients 


\section{FIGURE 1 Adjusted Exacerbation Rates in Patients with COPD on Compliant vs. Noncompliant GOLD Treatment Regimens (per patient-year)}

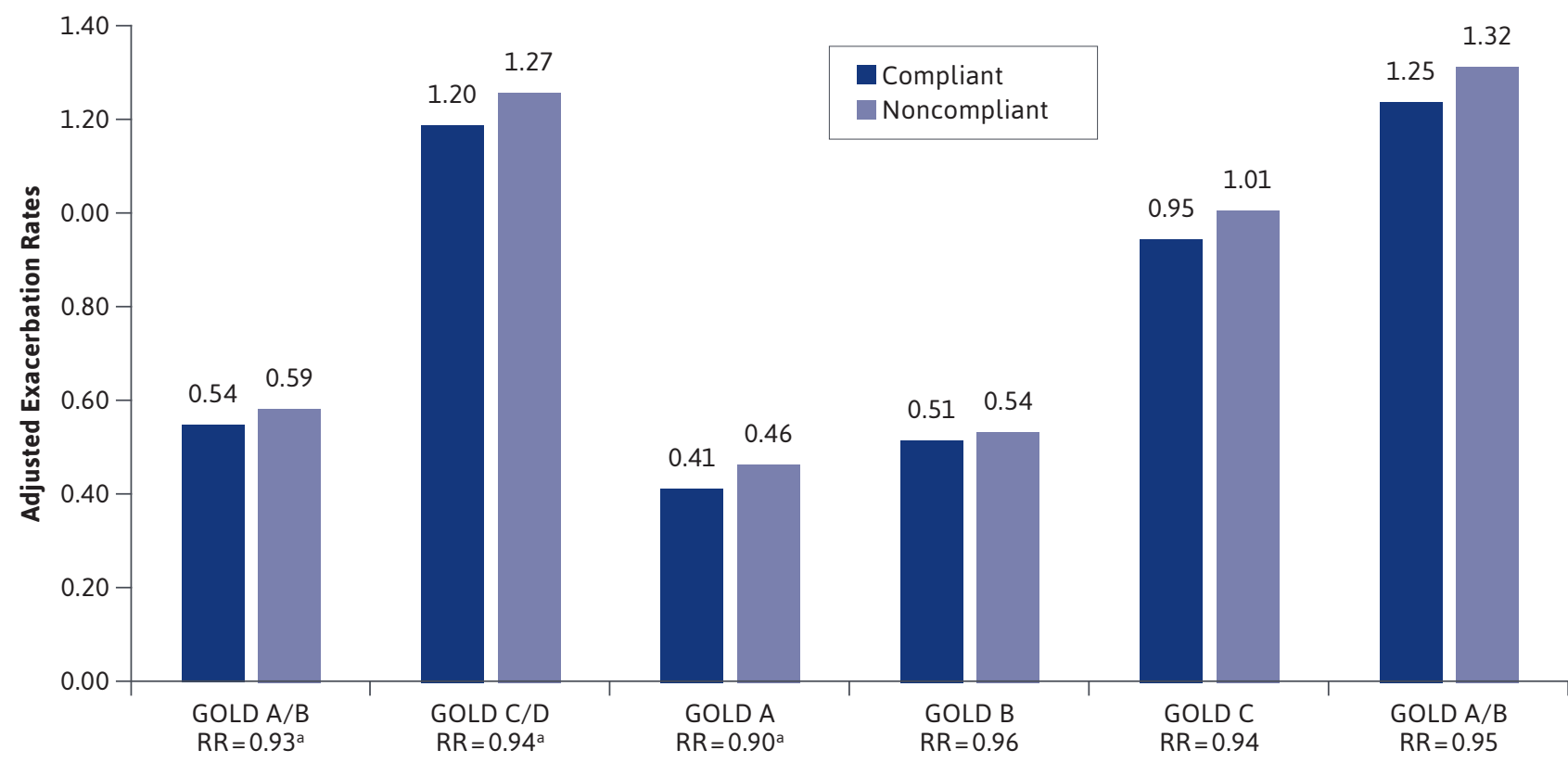

GOLD Risk Group

Note: $R R$ reference group is the noncompliant subgroup.

ap value $<0.05$

$C O P D=$ chronic obstructive pulmonary disease $; O L D=$ Global Initiative for Chronic Obstructive Lung Disease; $R R=$ rate ratio

with COPD did not receive a maintenance inhaler. ${ }^{15}$ Of note, the Make et al. study identified patients with COPD during 2004 and 2005 and the Diette et al. study in 2007 and 2008. Compared with our study's 2014-2017 time frame, there appears to have been some progress in increasing the rate of COPD maintenance therapy prescribing over the years, but more effort is still required to optimize these prescribing rates and improve patient outcomes.

We assessed the maintenance regimen for each patient with COPD with regard to the regimen's compliance with the 2017 GOLD prescribing recommendations. In the GOLD A/B and C/D groups, compliance with GOLD-recommended regimens was $32.9 \%$ and $58.9 \%$, respectively, with an overall compliance of $36.7 \%$. Individual GOLD A/B/C/D group compliance ranged from 25.7\% (GOLD B) to 61.5\% (GOLD D). This too corresponds very closely to previous research. ${ }^{8,16}$ Mannino et al. reported that $36 \%$ of patients with COPD were on a 2011 GOLD guideline-adherent regimen identified from a U.S. electronic health records database. ${ }^{8}$ Additionally, a large, multicenter, observational cohort study conducted between 2010 and 2016 by Ghosh et al. (2019) found that 52\% of the regimens for patients with COPD aligned with the 2011 GOLD recommendations, and only 37\% aligned with the 2017 GOLD recommendations. They also stratified their results by individual GOLD ABCD risk group and reported patients in the GOLD B group had the lowest alignment (14\%) while patients in the GOLD D group had the highest alignment (80\%). ${ }^{16}$ A consistent finding across studies that only slightly more than a third of regimens were compliant with 2017 GOLD recommendations suggests compliance with GOLD prescribing has continued to be suboptimal. For developing interventions aimed at improving prescribing compliance with GOLD, understanding the reasons behind these trends (e.g., suboptimal awareness and/or understanding of GOLD strategies, ${ }^{3}$ misclassification of COPD as asthma in general practice or consideration of asthma/ COPD coexistence,${ }^{17}$ medication access) need to be further investigated.

Inappropriate use of ICS-containing regimens, and in particular LABA/ICS regimens, were the most frequent reason for noncompliance to GOLD recommendations in our study. This is consistent with the findings of Ghosh 
et al., identifying LABA/ICS as the most common nonaligned regimen in their study. ${ }^{16}$ Of note, $90 \%$ of patients in our GOLD A/B group had a pharmacy claim for an ICS-containing regimen. This is worthy of highlighting as there is very limited evidence to suggest a benefit in these patients, coupled with the potential risk of pneumonia associated with chronic ICS use. ${ }^{18}$ In addition, studies also suggest that long-term ICS use is linked with an increased risk of diabetes or poor control of diabetes, bone density loss and fractures, cataracts, and mycobacterial infection, including tuberculosis. ${ }^{19,20,21}$ Indeed, in our study, GOLDcompliant regimens were associated with patient benefit in terms of lower exacerbation rates. Both the American Thoracic and the European Respiratory Societies have now released new recommendations on ICS use and withdrawal in $2020 .{ }^{22,23}$ Specifically related to ICS withdrawal, they provided a strong recommendation on continuing ICS regimens in patients with blood eosinophil counts $\geq 300 \mu \mathrm{L}$, and consider ICS withdrawal in patients with COPD without a history of frequent exacerbations conditional on providing treatment with 1 or 2 long-acting bronchodilators.

A unique aspect of our study is that we explored the association between regimens compliant with GOLD recommendations and outcomes such as COPD-related HRU, costs, and exacerbations. Consistent across all outcomes were documented clinical and economic benefits associated with GOLD-compliant regimens, especially in the more severe GOLD C/D group and, in particular, to the inpatient setting. As patients with severe COPD are traditionally associated with the highest clinical and economic burden, benefits in this population are noteworthy. These benefits were also observed within the GOLD A/B group, with less magnitude potentially due to lower overall intensity of care required. However, given the GOLD A/B group comprised $85 \%$ of the cohort, improved outcomes may have substantial population-level ramifications. Since exacerbations guide GOLD risk group classification and were modestly reduced in both the A/B and C/D groups, compliance with GOLD recommendations may potentially decrease the progression to and proportion of patients in the higher risk group. Overall, these data support the hypothesis that GOLD-compliant therapies may result in improved clinical and economic outcomes in real-world clinical practice.

\section{LIMITATIONS}

Our findings were subject to typical claims-based limitations, such as omissions, coding errors, and the inability to determine whether prescription fills were actually taken as prescribed by patients. Since the presence of a diagnosis code on a medical claim may be a miscode or a rule-out criterion and therefore does not guarantee positive presence of a disease, we attempted to mitigate this issue and the lack of COPD-confirming spirometry data by using a validated predictive model to identify the COPD study population that did not solely rely on diagnoses codes but also considered various demographic, clinical, and therapeutic characteristics.

Another drawback was the algorithm used to identify symptomatic burden has not yet been validated. We should also note that patients filling only rescue therapies or add-on treatments (e.g., xanthines and phosphodiesterase- 4 inhibitors) and not receiving any COPD maintenance medication were not considered for analysis. This may be reflected in our estimates of the individual GOLD risk group size, with potential overestimation of the GOLD $\mathrm{C} / \mathrm{D}$ population. Patient adherence to their maintenance regimens and subsequent escalation was also not evaluated. Assignment of medication compliance per 2017 standards for patients pre-2017 could have potentially misclassified their compliance status at the time of prescribing, but given minimal evolution of the recommendations, this provided the most applicable results for current treatment.

The generalizability of our results is limited to similar commercially insured/Medicare Advantage populations and may not be generalizable to other government-sponsored health insurance members or those uninsured/ underinsured who may not have access to the health care resources of interest. Finally, there is potential for unmeasured confounding (e.g., demographics, clinical symptoms, and lifestyle behaviors that effect disease progression and medication adherence) biasing the results.

\section{Conclusions}

The current study provides real-world insights into the association between compliance with 2017 GOLD treatment recommendations and COPD-related HRU, costs, and exacerbations. Suboptimal prescriber adherence to GOLD treatment recommendations continues to be prevalent with the primary driver of noncompliance appearing to be the overuse of ICS-containing therapies in patients with COPDprescribed maintenance therapy. Lower COPD-related medical HRU and costs were associated with compliance with GOLD treatment recommendations, especially within the inpatient setting. These data provide support for the premise that health care programs and/or interventions aimed at improving compliance with GOLD prescribing 
recommendations lead to beneficial clinical and economic outcomes in patients with COPD.

\section{DISCLOSURES}

This study was funded by Boehringer Ingelheim Pharmaceuticals, Inc. (BIPI). BIPI was given the opportunity to review the manuscript for medical and scientific accuracy as well as intellectual property considerations. Palli and Shaikh are employees of BIPI. Willey is an employee of HealthCore, which was contracted by BIPI to conduct this study. Zhou was an employee of HealthCore at the time of study execution.

Data were presented in part during an AMCP webinar (recording not made public) held in lieu of the Spring 2020 AMCP conference, which was canceled due to the COVID-19 pandemic.

\section{ACKNOWLEDGMENTS}

Editorial support and formatting assistance were provided by Elizabeth Apgar, $\mathrm{MPH}$, HealthCore, and was contracted and funded by BIPI.

\section{REFERENCES}

1. Srivastava K, Thakur D, Sharma S, et al. Systematic review of humanistic and economic burden of symptomatic chronic obstructive pulmonary disease. Pharmacoeconomics. 2015;33(5):467-88.

2. Global Initiative for Chronic

Obstructive Lung Disease. The global strategy for the diagnosis, management and prevention of chronic obstructive pulmonary disease. 2017. Accessed March 9, 2021. https://goldcopd.org/ wp-content/uploads/2017/02/wmsGOLD-2017-FINAL.pdf.

3. Global Initiative for Chronic Obstructive Lung Disease. The global strategy for the diagnosis, management and prevention of Chronic Obstructive Pulmonary Disease. 2020. Accessed March 9, 2021. https://goldcopd.org/ wp-content/uploads/2019/11/GOLD2020-REPORT-ver1.1wms.pdf
4. Make B, Dutro MP, Paulose-Ram R, Marton JP, Mapel DW. Undertreatment of COPD: a retrospective analysis of US managed care and Medicare patients. Int J Chron Obstruct Pulmon Dis. 2012;7:1-9. doi: 10.2147/COPD.S27032

5. White P, Thornton H, Pinnock H, et al. Overtreatment of COPD with inhaled corticosteroids-implications for safety and costs: cross-sectional observational study. PLoS One. 2013;8(10):e75221.

6. Palli SR, Buikema AR, DuCharme M, Frazer M, Kaila S, Juday T. Costs, exacerbations and pneumonia after initiating combination tiotropium olodaterol versus triple therapy for chronic obstructive pulmonary disease. J Comp Eff Res. 2019;8(15):1299-1316. doi: 10.2217/ cer-2019-0101

7. Palli SR, Frazer M, DuCharme M, Buikema AR, Anderson AJ, FranchinoElder J. Differences in real-world health and economic outcomes among patients with COPD treated with combination tiotropium/olodaterol versus triple therapy. J Manag Care Spec Pharm. 2020;26(10):1363-74. doi: 10.18553/ jmcp.2020.20159

8. Mannino DM, Yu TC, Zhou H, Higuchi K. Effects of GOLD-adherent prescribing on COPD symptom burden, exacerbations, and health care utilization in a real-world setting. Chronic Obstr Pulm Dis. 2015;2(3):223-35.

9. Palli S, Zhou S, Shaikh A, Willey V. Developing a claims'-based algorithm to identify US patients with COPD. Eur Respir J. 2019;54(Suppl 63):PA4431.

10. Macaulay D, Sun SX, Sorg RA, et al. Development and validation of a claimsbased prediction model for COPD severity. Respir Med. 2013;107(10):1568-77. doi: 10.1016/j.rmed.2013.05.012

11. Goossens LM, Baker CL, Monz BU, Zou KH, Rutten-van Mölken MP. Adjusting for COPD severity in database research: developing and validating an algorithm. Int J Chron Obstruct Pulmon Dis. 2011;6:669-78. doi: 10.2147/COPD.S26214
12. Wu EQ, Birnbaum HG, Cifaldi M, Kang Y, Mallet D, Colice G. Development of a COPD severity score. Curr Med Res Opin. 2006;22(9):1679-87. doi: 10.1185/030079906X115621

13. Zeiger RS, Schatz M, Dalal AA, et al. Blood eosinophil count and outcomes in severe uncontrolled asthma: a prospective study. J Allergy Clin Immunol Pract. 2017;5(1):144-153.e8. doi: 10.1016/j. jaip.2016.07.015

14. U.S. Bureau of Labor Statistics. Consumer Price Index. 2017. Accessed March 23, 2021. https://data.bls.gov/ cgi-bin/surveymost?su

15. Diette GB, Dalal AA, D'Souza AO, Lunacsek OE, Nagar SP. Treatment patterns of chronic obstructive pulmonary disease in employed adults in the United States. Int J Chron Obstruct Pulmon Dis. 2015;10:415-22. doi: 10.2147/COPD.S75034

16. Ghosh S, Anderson WH, Putcha N, et al. Alignment of inhaled chronic obstructive pulmonary disease therapies with published strategies. Analysis of the Global Initiative for Chronic Obstructive Lung Disease Recommendations in SPIROMICS. Ann Am Thorac Soc. 2019;16(2):200-08.

17. Miravitlles M, Garcia-Sidro P, Fernandez-Nistal A, Buendia MJ, Espinosa de los Monteros MJ, Molina J. Course of COPD assessment test (CAT) and clinical COPD questionnaire (CCQ) scores during recovery from exacerbations of chronic obstructive pulmonary disease. Health Qual Life Outcomes. 2013;11:147.

18. Kew KM, Seniukovich A. Inhaled steroids and risk of pneumonia for chronic obstructive pulmonary disease. Cochrane Database Syst Rev. 2014;(3):CD010115.

19. Brode SK, Campitelli MA, Kwong JC, et al. The risk of mycobacterial infections associated with inhaled corticosteroid use. Eur Respir J. 2017;50(3):1700037. doi: 10.1183/13993003.00711-2017 
20. Loke YK, Cavallazzi R, Singh S. Risk of fractures with inhaled corticosteroids in COPD: systematic review and meta-analysis of randomised controlled trials and observational studies. Thorax. 2011;66(8):699-708. doi: 10.1136/ thx.2011.160028

21. Suissa S, Kezouh A, Ernst P. Inhaled corticosteroids and the risks of diabetes onset and progression. Am J Med. 2010;123(11):1001-06. doi: 10.1016/j. amjmed.2010.06.019
22. Nici L, Mammen MJ, Charbek E, et al. Pharmacologic management of chronic obstructive pulmonary disease. An official American Thoracic Society Clinical Practice Guideline. Am J Respir Crit Care Med. 2020;201(9):e56-e69. doi: 10.1164/ rccm.202003-0625ST
23. Chalmers JD, Laska IF, Franssen FME, et al. Withdrawal of inhaled corticosteroids in COPD: a European Respiratory Society guideline. Eur Respir J. 2020;55(6):2000351. doi: 10.1183/13993003.00351-2020 